\title{
Editorial to Special Issue INTUITION
}

\author{
Nadia Magnenat-Thalmann • Angelos Amditis • \\ Ioannis Karaseitanidis
}

Published online: 20 August 2008

(C) Springer-Verlag 2008

This special issue contains a selection of papers presented at the 4th INTUITION International VR/VE Conference. The papers selected in this special issue have been reviewed by the INTUITION conference program committee members and the papers reflect experimental work in the field of AR and VR. INTUITION is one of the largest European Networks of Excellence dealing with VR and AR in Europe and the readers will surely appreciate to read some work being developed in this area.

Nadia Magnenat-Thalmann

Editor-in-Chief

The Visual Computer

The purpose of this Special Issue is to present some of the new developments as they were discussed at the 4th INTUITION International VR/VE Conference and Workshop held in Athens in October 2007 (for more info check www.intuition-eunetwork.org). A scientific committee selected the most interesting and well prepared papers, out of the 24 peer reviewed papers, presented at the conference for inclusion in this Special Issue.

The technology per se is a multi-disciplinary field encompassing different scientific and technological attributes. This fact makes strategic research planning and technology

\section{N. Magnenat-Thalmann $(\bowtie)$}

University of Geneva, Geneva, Switzerland

e-mail: thalmann@miralab.unige.ch

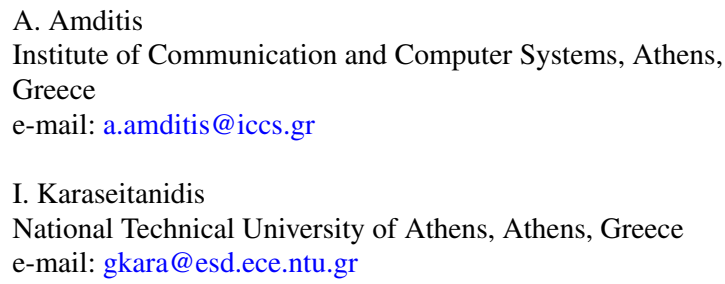

know-how acquiring a difficult task. That is why the first paper focuses on studying the future research perspectives and the means to structure knowledge and resources in VR within Europe. The other papers are addressing issues from interfaces and interactions up to robotic and industrial applications.

The paper by W.A. Aprile et al. is focusing on the construction of a tracked robotic head (driven by joystick) to provide switching stereo/mono vision.

Interactive simulation in automotive design is being addressed by L. Nikitina et al. who describe also how to build large-scale 3D virtual mockups.

Speaking of interaction techniques, a very important subject for VR, L. Vanacken et al. present a method for speech grammar generation that can be combined with interaction techniques aiming at easing the development process of VE applications.

C. Syllebranque and S. Boivin present a method enabling to estimate the mechanical parameters of soft bodies directly from videos of solids getting deformed under external user action.

Continuing with haptics, a very important interaction technique for VR/AR applications, R. Iacob et al. speak about assembly/disassembly simulation of industrial products using haptic devices.

Emotion experiences could not be missed in a discussion about interactions, so $\mathrm{N}$. Zagalo et al. present an emotion module from an authoring tool of interactive storytelling.

Moving now to applications, R. Reif and D. Walch discuss augmented and Virtual Reality applications in the field of Logistics.

Finally, D. Harm et al. study the after-effects of exposure to Virtual Environments taking into account duration and exposure rates.

Dr. Angelos Amditis

Dr. Ioannis Karaseitanidis 